\title{
Research on Construction of Modern Intelligent Community Based on Internet of Things \\ ZHOU Huachun ${ }^{1, a}$
}

\author{
${ }^{1}$ Department of the internet of things ,Chongqing College of electronic engineering, ChongQing, \\ 401331, China
}

a420749294@qq.com

\begin{abstract}
Keywords: Internet of Things, Intelligence Community, ZigBee
\end{abstract}
\begin{abstract}
With the introduction and development of the concept of things, intelligent residential quarters ushered in a new development opportunity. Intelligent residential quarters combined with networking technology to enhance their capabilities to make intelligent residential quarters as a node on the wisdom of the city. The collected information to the Intelligent System of Things platform can achieve a higher level of unified management and information sharing. In this paper, the overall design of intelligent residential networking technology, Internet of Things technology for the intelligent plot to do some exploration model for example, there are certain practical significance and practical value. The main results are as follows: First, the overall design, based on the top-level design thinking centralized supervision, design, a set of data-aware layer, network transport layer, middleware layer, four-layer data processing system Intelligent Community System structure in this each subsystem can be under the framework of the intelligent community scale.
\end{abstract}

\section{Theoretical Introduction of Internet of Things}

The definition of things: The radio frequency identification (RFID, infrared sensors, global positioning systems, laser scanners and other information sensing device, according to the agreed protocol, to any article connected to the Internet, information exchange and communication in order to achieve . intelligent identification a network positioning, tracking, monitoring and management of a deeper understanding of things, first of all, networking is an extension and expansion of the Internet, the Internet is still the core and foundation; secondly, extension and expansion of its client to any goods and goods between the exchange of information and communication.

Internet of Things "upgrade", although many have already started to develop technology, but the technology is very comprehensive and strong. For such systems engineering Intelligent Community, require the participation of all walks of life, is the need to integrate different technologies or product system, which is downstream of the close cooperation on the networking industry chain results. RFID is responsible for storing the information of the object tag embedded in the object, for identification; analog identification (various sensor devices) of various sensors using real-time dynamic physical analog information into digital information for transmission to the network ${ }^{[1]}$.

Network is a channel for transmitting information through a variety of wireless networks (such as Wifi, Bluetooth, ZigBee, GPRS, etc.) and cable networks (such as the Internet, fixed-line networks, smart grids, etc.) to transmit digital information to the background processing system. Intelligent processing chain refers to the use of cloud computing platforms Mass secure storage and intelligent information processing characteristics of the upstream transmission of huge amounts of data to be stored and processed. Applications link through the carrier platform to carry out application of all walks of life, it will extend laterally from the vertical industry applications, mass data processing and information management needs will give rise to a higher demand for operators. Intelligent Community is a relatively independent of the inner city area, unified management, intelligent building residential quarters feature similar residential buildings. 


\section{Internet of things Requirements Analysis}

Intelligent Community integrated computer technology, communication technology, control technology and IC card technology, the use of system integration, and gradually set up a household and tenants, tenants and community service centers and multimedia integrated information platform for residents and outside the community, and provide tenants with a safe, comfortable, convenient, energy efficient living environment. Intelligent System is a modern high-tech of product and technology in the residential area, which mainly include security subsystem, information management and monitoring subsystem and network subsystem ${ }^{[2]}$. Fig.1 shows Internet of things requirements analysis.

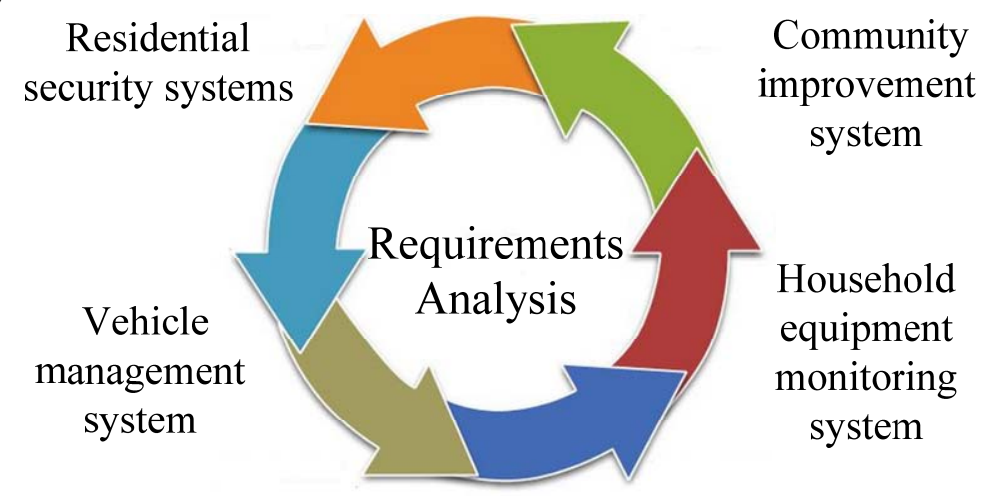

Fig. 1.The Internet of things requirements analysis

The development of modern residential area is safe, comfortable, fast, convenient and secure community which is particularly important. An integrated intelligent security systems including access control systems, video surveillance systems, intrusion alarm systems and electronic patrol system. Through a variety of alarm detectors to form a three-dimensional protective space; patrol system to strengthen the efficiency of air defense, security personnel must ensure that the designated time patrols in place. The emergence of the Internet of Things technology, different cells can be integrated into the security subsystem networking platform, not only within the cell can be achieved unified management and intelligent monitoring, in turn, can also achieve unified management and unified the whole city.

Appliances home life is an important part, after these appliances intelligent control, will bring more intelligence and quality services to our lives. Technically, white goods by motor converts electrical energy into heat energy, kinetic work, belonging to the control network intelligent home network is home inside the device, so as to form a unified smart home network, as part of the Internet and mobile communications world the purpose is to enable users to easily remotely monitor and control your home equipment, to provide users with convenient services, through mobile phones, the Internet remote home control and management. Digitized medical services based on community health The Internet of things, telemedicine services to patients provides a convenient, eliminating the patient back and forth between home and hospital laboring to improve the health level of intelligence ${ }^{[3]}$.

\section{Overall System Design of Intelligent Community}

Prior to the overall design, top-level design departure from the system, to determine the overall design goal: deploy Intelligent Community centralized monitoring and management system, the use of computing and storage capacity Internet cloud platform for residential property owners to store summary information on security situation centralized monitoring of all types of property management business for unified management via the Internet in order to achieve centralized management of the intelligence community. For smart cell structure, we propose the use of research data Status introduction perception layer, network transport layer, middleware layer, data processing 
and management of four architectures. Fig.2 shows intelligent community platform.

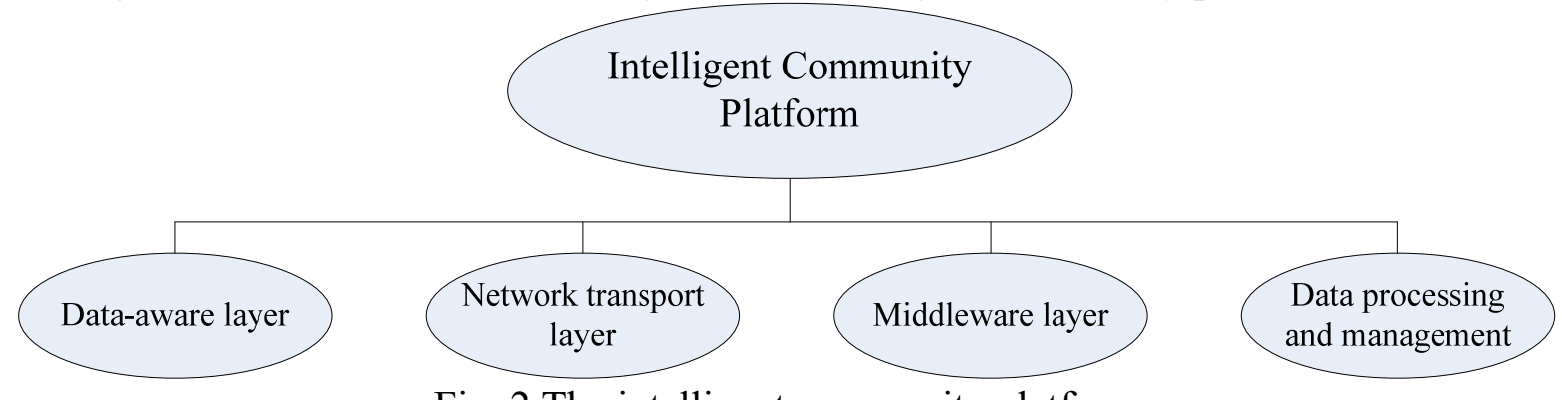

Fig. 2.The intelligent community platform

The households of smart home and intelligent community T-pad centralized monitoring and management system via LAN communication cell, architecture, local network can be a client / server architecture can also be used browser / server. In the C / S architecture, the client and the server need to design peer software architecture. In the B / S architecture, the intelligent home and intelligent community monitoring terminal centralized monitoring and management system, using HTTP port ( 80 for communication. The various operations concentrated eleven intelligent community centralized monitoring and management system terminal processing in the server side, intelligent community centralized monitoring and management system as a user terminal, only need to install the WEB browser.

Intelligent Community centralized monitoring and management system using three-tier architecture model of software design, namely WEB server, APP server, DB server, WEB server for residential property owners, the use of cell centralized monitoring and management system. On the one hand, we can provide a full range of information services for the property management, water, electricity, coal, fire and other aspects of property management information; on the other hand, the use of centralized monitoring and management system, monitoring and alarm functions, promptly notify owners and rushed to the accident scene, secure management of information technology. Intelligent Community centralized monitoring and management system to give full play to its own computing power and a huge database of resources, a variety of technical and scientific research and development ${ }^{[4]}$.

\section{Internet of Things Practical Application}

The Internet of things technology for intelligent residential quarters, the collected information to achieve a higher level of unified management and information sharing, enhance the intelligence community's own features. Past the parking management system billing charge management functions as the focus for security, operational efficiency and human aspects parking not considered comprehensive. Fig.3 shows intelligent vehicle management system.

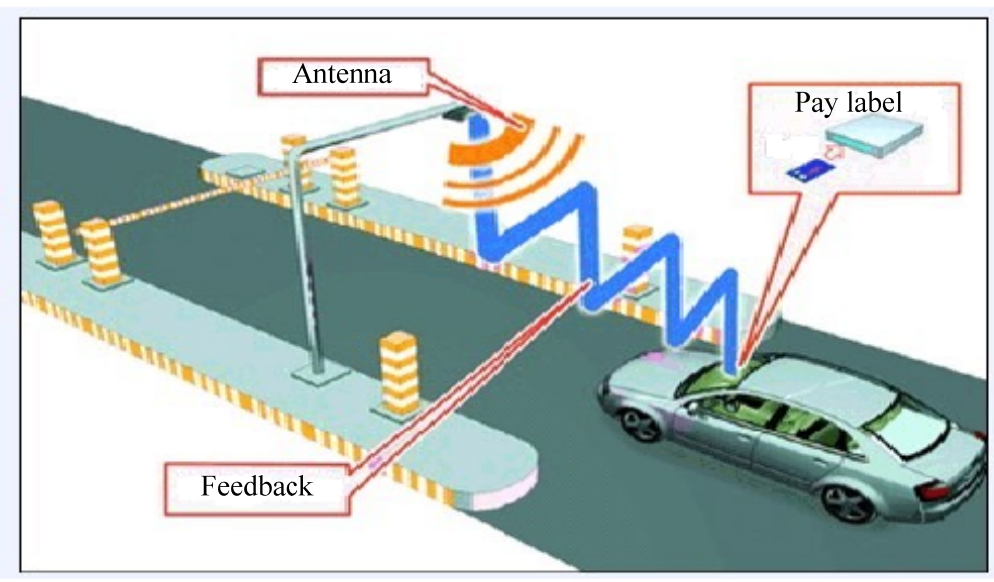

Fig. 3.The intelligent vehicle management system

Based things related technologies area intelligent vehicle management system that uses 
advanced RF technology, combined with video recognition technology for unmanned payment, transparency of information, real-time, can reduce the payment time, save manpower and financial resources, and ultimately intelligent traffic management of. Electronic toll collection system is one of the service functions of intelligent transportation system, passing vehicles through the crossing without stopping, that can automatically charge. Database design principles: to ensure that the needs of low complexity of the program, the application must meet the system requirements of universality; ensure the accuracy of the data, effectively control redundancy to improve database performance; all data tables are included in this database, the reduction the correlation between the data in the database table ${ }^{[5]}$.

Intelligent Vehicle Management System is simple, convenient, advanced features and other characteristics, using the shortest time to enter or leave the parking lot, avoiding the red tape, improve the quality of management, access to good social and economic benefits. Things cell vehicle management system by the background system, lane controller, speed sensor devices and microwave communications equipment and other components. System contains the following sections: Vehicle identification and radio frequency signal processing section, video surveillance and image transmission section, intra-regional wireless sensor network part, GPRS communications section, integrated decision-making part.

\section{Conclusions}

The Internet of things applications era of intelligent residential quarters of view, research networking technology in the application of intelligent residential quarters, proposed the use of networking technology to the residential area of equipment and systems solutions and implementation methods for monitoring and unified management, final things platform to achieve elevator remote monitoring and management system based on. Things technology use the residential area to enhance the functionality of intelligent residential system, enrich the connotation of the Intelligent Community, so that residential areas as a node on the wisdom of the city, in the promotion of smart city, safe city construction has theoretical and practical value. Intelligent Community designed four architectures: data perception layer, network transport layer, middleware layer, data processing and management, and applied separately for each level sensor technology, fuzzy control technology gives smart home system design and analysis.

\section{References}

[1] J. K. W. Wong, H. Li, S. W. Wang. A review research on automation in construction of intelligent building [J]. Artificial Intelligence Research, 2011, 14(4): 143-159.

[2] R. Agraw, A. Vinei. A service-oriented architecture for rapid development of web application[J]. Computer Networks, 2011, 5(39): 86-91.

[3] W. G. Beazley, Framework for information technology integration in building and related industries [J]. Computer Networks, 2012, 7(31): 143-146.

[4] Campbell, Mac Gregor. Build your own Internet of things [J]. New Scientist, 2012 214(2861):44-47.

[5] H. Y. Huang, J. Y. Yen, S. L. Chen. Development of an intelligent energy management network for building automation [J]. Automation Science and Engineering, 2012, 1(1): 14-25. 